1I. Derma -1. Ground Connective Tissue. a. Papillæ flattened. b. Tissue of floor of blister almost homogeneous. 2. Capillaries. $a$. Contraction of capillaries. $b$. Contraction of endothelial cells. c. Coagulation and fibrillation of contents. 3. Nerves. Remarkable distinctness of Meissner's corpuscles.

III. Panniculus Adiposus.-1. Ground-tissue unaltered. 2. Fatlobules unaltered. 3. Sweat-glands. Fusion of cells and fibrillation af contents of ducts near surface of cutis vera. 4. Nerves. Doubtful swelling of endoneurium. 5. Vessels. Doubtful excessive contraction of arteries.

The recorded morbid appearances of death from lightning are noteworthy in general, on account of the absence of obvious internal lesions ; in this they agree with the present case. Although at least two fatal cases have been reported in this country from the action of the electrical fluid generated by machinery, yet we are not aware that the pathological changes have been placed on record.

Looking at the present increase in the application of electricity to various purposes, it seems quite probable that fatal accidents will increase among those who, from the nature of their vocation, are daily engaged among the complicated machinery. Important medicalegal questions may arise on the subject of a person found dead in the noighbourhood of electrical machines or conducting wires. So, too, it is sufficiently obvious that such an agent as electricity might be employed by murderers acquainted with the working of electrical apparatus, and with the absence of traces of violence left by the action of the current. It may, therefore, justly be said that a record of such cases is of importance, if only to serve as references for future comparison,

From the evidence of those who stood near, it would seem as though the man were instantly deprived of life. No doubt the vital spots at the base of the brain are, in such cases, markedly implicated. The fluid condition of all the blood, and the uncontracted state of the heart, were quite striking, but the naked eye appearances of injuries like these are sure to be obscure and negative in character, and it is only by the microscope that we can hope to appreciate any of the minuter changes that occur. The condition of the blistered skin is very peculiar, and differs from that of the ordinary burn-blister in several particulars. Such appearances would be quite recognisable on another occasion.

\section{THE PREVENTION OF EPIDEMICS.}

Read in the Section of Public Medicine at the Anrual Meeting of the British Medical Association.

By FZRA M. HUNT, M.D., New York.

IN this brief paper, I only attempt a syllabus of the method in which we should conduct our inquiries in order to accomplish more in the prevention of epidemics. In dividing up and assigning this study of epidemics, the following are the chief inquiries and observations to be made.

1. What is the contagium vivum? As to this, we have to deter mine in what its entity consists ; whether it be particulate or gaseous, whether it be so specific and singular in its character that it is always the same as to quality or quantity; or, if capable of modification, how it can be modified; whether it be always derived, or whether it be produced so as to be in a sense spontaneous. In a word, we must study the pontagium; 80 as to know the most possible about it in its own individuality.

It is almost peedless to say that the germ-theory of disease has immonsely broadened this part of our study; but perhaps it is needful to say that the fact that we are probably nearer than ever before to the identification of certain or most eontagia, does not clear up the question as to whether they are derived or spontaneous, or how the benign bacillus becomes malign; it does not settle their origin, and so as yet does not, by original dealing with the entity, assure us how we can prevent its existence, and so radically prevent epidemics

Most contagia thus far seem identified with plant-life. We may get lessons as to modes of study from the fact that many communicable diseases seem to have to do with plant-life.

It is not surprising that Hallier, Panum, De Bary, Cohn, Thomé, Darwin, and others who were early in these studies, were botanists and it has seemed to us that we would have done well, in our study and classification of this infinitesimal life, to have followed still more alosely in their methods. The botany and zoology of parasitic microseopic life will yet have a classification well nigh as extended as that which belongs to life in its more visibie froms.

The worker in this field is in a garden of contending vegetations, in which it is not enough to say that each spore or germ or seed will produce its kind. Omne ovum ab ovo is true, enough in animal life, but this does nat prove the impossibility of a mule, or of some other product, or of a disease equally unique in its way. It is a marvel to see how the same seed can be so cultured that its products may be very greatly varied, and how marvellous are the hybrids or sports that may result. To me, the views of Pasteur do not seem to conflict with those of Bastian, nor does the doctrine of a result in disease, so modified as to almost defy identification, or so crossed as to give a mongrel, or what practically as to treatment is new, at all lean to the doctrine of spontaneous generation, as formerly taught. Cholera, typhoid fever, diphtheria, etc., some day become existences amid intense disturbances of natural processes. Although now generally occurrent from derived source, this does not preclude the possibility of their occurrence locally and sporadically, without an antecedent case - the only antecedent being the same intense disturbance of natural processes. It is strange that nitric acid and glycerinethe one corrosive, the other emollient-should have been so long handled in the laboratory before nitro-glycerine should have begun to exist. So, from special relationship of ordinary filth and extraordinary atmospheric conditions, there probably have come, and will come, new diseases, the causation of which we do not define when we find a germ, however much it may aid us in diagnosis.

II. Our next study, although allied, is quite distinct. Failing in finding the seed or the contagium, or destroying it, how shall we make of its vivum a case of suspended animation? How shall we sterilise it ? I will not here discuss its culture in order to enfeeble it, as this is going on well exough. But I allude to the study of how we may provide it a sterile soil, either in the surroundings or in the person. This involves a close study of the habits of each contagium, on what it flourishes best without or within.

While the name filth-diseases is a convenient generalisation, we are not exact in our study until we accurately define decompositions, putrefactions, associated animal or plant life, so as not only to affirm, for instance, that vegetable decay causes periodic fevers, and animal excretion typhoid fever, but also to carry the details of observation, of facts, and of experiment, to an extent which shall enable us to approach the exactness of the botanist, who says that the silkworm thrives best on the mulberry, and that the potato-bug has an especial relish for the egg-plant. For the prevention of epidemics, there is this special field of study as to all the minute canditions or surroundings outsiae the body. We shall have gained very much when these scientific or expert methods, which are applied by the skilful naturalist, for instance, to all harmful or poisonous plants or insects, are applied to all embarrassments to our lives productive of disease as found outside and about us.

While the first plan, that of discovering a germ, is radical, yet it is not necessarily indispensable, if we can so apprehend the necessary conditions for propagation as to circumvent these. Hence students of this second class are not discomfited, even if the first study be incomplete.

III. The study of the individual in his relation to the contagium vivum, and to his surroundings, is another distinct study, inviting to another class of skilled observers-a laboratory in which minute work is greatly needed.

1. Under what circumstances does a human being come to be the host of something inimical to him, and prepared for an invasion of something, perhaps, producing disease? It is not enough to say of it that it is its nature to seek or to be communicated to human beings, and to develop into a disease. The fact that some persons, without having had a disease, are proof against its invasion is a significant one, and worthy of great inquiry to those who would like to put all in the same resistful condition. The fact that once having had some diseases protects most from them afterwards, or does not protect some, or, while protecting all, does not protect all for an equal length of period, cannot but make the epidemiologist very inquisitive to find out the reason, and so put it into effective preventive operation. Watson and others have stated that it is because the disease exhausts its neces. sary pabulum in the system; but, if this: be so, the statement is in. complete, until we find out what pabulum it has wasted and what it has exhausted. As in the old system of inooulation it was discovered that, by diet or certain preparative treatment, small-pox could be modified in its virulency, and the secondary fever aborted, the why and the wherefore ought not to be given up by the modern medical profession, as it apparently had to be in the former.

2. When, too, we find that, as in inoculation to prevent pleuropnemngoia in cattle, we secure an inflammatory action, and a constitational effect through a mascle, and so prevent the fatal attreck on a vital organ hilke the lung by a sort of inrtificiad netastasis, we need to 
study how much of diversion and limitation of epidemics can be secured by their artificial and preliminary introduction into some part other than that which it seems their habit to attack with virulence. How much of the modified effect is owing to mode of introduction rather than to attenuation?

3. Still, further, if changes can so be wrought in systems as to make them unreceptive of diseases, as we know to be the fact with many ailments, cannot and do not quinine, alcohol, potassium-chloride, ferric chloride, and other antimicrophytes, antizootics, or antiseptics, cause the blood and tissues to be protected from the invasion while there is exposure thereto, and so may we not prevent epidemics?

We now know, by actual experiments and observation, that we can see the blood-corpuscles multiplying during the administration of iron, and can, with small doses of quinine, potassium-chloride, arsenic, etc., have the sustained presence of these in the blood. There is good reason for thinking that, during such presence, the blood and tissues become resistful to that multiplying plant-life which, either directly, or by its overpowering abundance, or by mechanical clogging of blood-paths, constitutes the gravity of the disease ; also that we can anticipate the action of the introduced contagium, and make the system refuse to nourish or propagate the parasite. Thus, either all may escape the prevailing influence, or so many that it cannot prevail among the people, and so cannot become epidemic. This temporary prophylaxis, during what, for the want of a better term, has sometimes been called an epidemic tendency or constitution of the atmosphere, is most worthy of accurate trial. As to its reality, many corroborative facts from Polli, Parkes, Panum, Bert, etc., can be adduced.

Then, last of all, comes the question of the limitation of diseases in their attempt to become epidemics, and after they have so become; the former being most valuable forethought, the latter being not unimportant afterthought. This limitation involves the study of the natural history of every communicable disease in all its minutiæ, that we may know its times and seasons, the distance at which it can be propagated, the length of period of its communicability, the secretions or families most likely to convey it, the relation of breath and of air to it, and all other facts which are relative to its transmissibility ; and, as a sequel, comes in a study of isolation, and of disinfection, etc., as a system of rules and regulations.

Our imperfection of knowledge does not hinder us from general rules and methods founded on apparently correct generalisation as to all communicable diseases, and specifications as to some, so far as we know.

The most inspiring result of what we may call the modern departure in epidemiology, is not so much the conclusiveness or completeness of facts in any one direction as the unmistakable indication of precision in the laws of communicable diseases, these heing as accurate as those that obtain in nature, giving us the comfortable persuasion that they are ascertainable and classifiable, although, for various reasons, difficult of ascertainment, but likely to yield to analytic and statistical methods, and to that tact of experience in observation which can be acquired but cannot be described.

The chief contentions of this paper are, therefore, as follows

1. In the study of the contagium vivum, we are to recognise not only change from culture or attenuation, but, as in plant-life and animal life, to recognise manifold changes which may take place, so discursive as to obscure identity, and so as lo make what, in pathology and treatment, may be a new disease, without involving the doctrine of spontaneous generation.

2. We must give significance to the effect of imparting a disease to the system by channels or modes of introduction different from what may be called its normal method of entrance, and allow for modification of effect from this cause, without any real attenuation.

3. We must study closely, not only the general effects of surround. ings, but the fertilisation or rankness which certain diseases attain from a compost especially adapted to them.

4. We need, with the same precision, and in a similar direction, to ascertain what are the conditions of individuals who furmish in themselves extraordinary soil for communicable diseases, or who withstand seizure amid exposure, or have but a mild attack, and to recognise that there are ascertainable reasons for this difference, a definite law of susceptibility.

5. We need to give great prominence to a study of direct prophy. lactic methods, and such as shall seek, during exposure or the prevalence of an epidemic, to prevent an attack, by imparting to the blood and tissues the presence of such substance as shall prevent those changes which an introduced morbific agent would otherwise set up.

Medical Magistrate, -C. J. Westrop, M.D., of Carrow Carlin, has boen appointed a Justice of the Peace for the Co. Fermanagh.

\section{NARRÁTIVE OF AN INSTANCE OF CURE OF LUPUS ERYTHEMATOSUS.}

BY JONATHAN HUTCHINSON, F.R.S., Emeritus Professor of Surgery to the London Hospital.

ALL who know the disease will, I am sure, admit that cases of recovery from lupus erythematosus are, unfortunately, rare. As an instance of completed cure, the case which I am about to relate becomes, I think, of much interest.

I first saw Mr. J. P. B. on March 4th, 1881. He came to me on account of patches of erythema-lupus on each side of his nose, about its middle. There was a third on its ridge, near the tip, but the three did not coalesce, and thus the bat's-wing was not complete. Their arrangement was, however, quite symmetrical. Mr. B. was a tall man, rather spare, but in fairly good health. His age was 45 . He had never suffered from actual chilblains, but had a feeble ciroulation, and dusky ears. A maternal aunt had died of phthisis. He had himself once consulted the late Dr. Baly, in the belief that his chest was delicate, but had been told that he ailed nothing but " dyspepsia and weak heart." His skin had always been very irritable, and he was liable to little spots on the hands, etc., which itched intolerably, and which he used to scratch until they became sore. Such was his state when the erythema-patches showed themselves on his nose. I may add that he was living the life of a country gentleman in a cold district. The patches had been present about a year when he came to me. I prescribed for him arsenic internally, and a weak lotion of $\operatorname{tar}$ and lead to bathe the patches.

Between 1881 and April 1883, I saw nothing of Mr. B. At the latter date, his surgeon, Mr. Williams, of Norwich, wrote me that he was worse. It was now decided to insist on the use of arsenic.

In February 1885, Mr. B. called to show himself, and to tell me that his lupus was quite well. It was absolutely so. White, thin, inconspicuous scars took the place of the former patches, and there was neither thickening nor erythema at their edges. The scars on the sides of the nose were each as large as a shilling, that on its middle not so big. On the scalp, which was nearly bald, there were several other scars as big as the end of one's thumb, which were the remains of other patches which had developed since his visit to me. As regards his cure, $\mathrm{Mr}$. B. said that it was unquestionably due to arsenic. He said that, in consequence of my having remarked that I trusted most to external treatment, he did not, on the first occasion; continue the arsenic, having a prejudice against it, but used the tar and lead wash assiduously. The patches increased, and new ones on his scalp formed. Two years later, in $1883, \mathrm{Mr}$. Williams insisted on his taking the arsenic, and increased the dose. It was continued for fifteen months regularly, caused a sharp attack of shingles, and made the eyes red and irritable, but, in the end, quite cured the lupus, I inquired carefully as to whether any local remedy had been employed simultaneously, which might have been the real agent in the cure, but it did not appear that such was the case. As regards the patches on the scalp, it is true that Mr. B. thought that a hair-wash, "which made the scalp smart," had done them good ; so much impressed had he been with this belief, that he applied the wash to his nose also, but this had been done only on a few occasions, and had not, he thought, helped the cure. It must also be remembered that he was taking arsenic all the time that the wash was being credited with, the cure of the scalp-patches.

Comments. - I have prescribed arsenic for many other cases of lupus erythematosus, but, having never realised any definite result, I have not urged it with much faith. It may easily be the fact that it has seldom been sufficiently pushed. It will be seen that; in this case, we have proof, in the occurrence of arsenical shingles and of red eyes, of the full physiological influence of the drug. .. I have, in a certain minority of cases, cured, or partially cured, this disease by the use of external applications, hut have never thought that internal medication had any definite effect. This case would certainly suggest a more free use of our great remedy. I fear, however, that we shall find that it is by no means generally successful, and that Mr. B.'s case is, after all a fortunate exception to rule. I have certainly seen many cases in which other surgeons had, before the patient came to me; pushed arsenic very freely, and sometimes apparently rather with injury than with benefit. The clinical fact that lupus erythematosu's differs from all other forms of lupus in its tendency to develop symmetrically in isolated and independent patches, seems to show an alliance with psoriasis. At any rate, it indicates a constitutional rather than a local origin, and, as such, implies the probable need of internal remedies. 